\title{
Perceptual cues used in reproduction of inspired volume
}

\author{
LAWRENCE J. FOLINSBEE, JEFFREY A. GLINER, and STEVEN M. HORVATH \\ University of California, Santa Barbara, California
}

\begin{abstract}
Man is capable of reproducing an inspired lung volume (IV) with relative precision ( $\pm 3 \%-8 \%$ ). To investigate the importance of cues which may be used to determine IV, we performed two experiments. In each, the subject inspired a "standard" breath (from resting end-expired lung volume) corresponding to $25 \%, 50 \%$, or $75 \%$ of inspiratory capacity (IC) and then expired nearly completely. From this expired volume, the subject either tried to reproduce the tidal volume (i.e., volume of air inspired) of the first breath (volume reproduction) or to reproduce the previous end-inspired lung volume (position reproduction). The constant error indicated that subjects overestimated tidal volume by about $200 \mathrm{ml}$. Despite this overestimation, the variability of reproduction was less for volume reproduction than for position reproduction when IV was small. The JND for volume reproduction increased linearly with increasing IV, whereas the JND for position reproduction was similar at all IVs so that the Weber ratio decreased. These observations suggest that, in the perception of inspired volume, the sensation of end-inspired position is important when the inspired volume is large, but that subjects apparently rely on some other cue to reproduce small lung volumes accurately.
\end{abstract}

Man's ability to perceive and control lung volume consciously is well known. However, the different techniques utilized to assess the accuracy of matching lung volume have led to considerable variation in results. Furthermore, most of the research in this area has focused on sensitivity to changes in lung volume, while little attention has been given to the cues that allow these changes to be detected.

A number of cues could be used to reproduce lung volume accurately. First, the individual could exert a given inspiratory muscle force and aim to reproduce precisely the duration for which it was applied, an "impulse-timing" approach that requires no sensory feedback of the end point of the movement. Second, the individual could assume that the volume at which each inhalation began (the same volume at which the previous exhalation ended, referred to as end-expired or initial volume) was consistent from breath to breath and then aim to reproduce the same end-inspired or final volume. A third and somewhat more complex procedure would be for the individual to gauge the magnitude of both initial and final volume and then perform a sensory subtraction procedure to estimate volume difference. (We view this latter possibility as somewhat unlikely.)

This work was supported in part by National Institutes of Health Grant NIH HL 26034. The technical assistance of Colleen McCary is gratefully acknowledged. Correspondence and reprint requests should be addressed to Lawrence J. Folinsbee, Institute of Environmental Stress, University of California, Santa Barbara, California 93106 . The present address of Jeffrey A. Gliner is Department of Occupational Therapy, Colorado State University, Fort Collins, Colorado 80521.
In this study, we examined the second of these possibilities-that subjects use cues to end-inspired volume in reproducing lung volume. In normal breathing, end-expired volume remains relatively constant (Gliner, Folinsbee, \& Horvath, 1981) and thus permits accurate volume reproduction based on the end-inspired volume of the standard breath, since the tidal volume (end-inspired minus endexpired) would then be the same. That relationship was changed by having the subject exhale fully after the standard breath, thus altering the end-expired volume that initiated the reproduction breath. In one condition, the subject was asked to reproduce the tidal volume of the standard breath; in another condition, he was asked to reproduce the end-inspired volume of the standard breath. If end-inspired volume is the dominant cue normally utilized in volume reproduction, performance in the first condition should be poor compared with that in the second.

\section{METHOD}

\section{Subjects}

Four male and three female volunteers from our laboratory staff served as subjects; the nature and purpose of the study were explained to each subject. Vital capacity of the subjects ranged from 3,971 to $6,284 \mathrm{ml}$ (mean $=5,058 \pm 847$ ); inspiratory capacity ranged from 2,550 to $3,735 \mathrm{ml}$ (mean $=3,218 \pm 552$ ) (see Figure 1 for definition of lung volumes). The subjects had had previous experience with respiratory apparatus and were given several practice trials.

\section{Procedure}

We used a modification of the method of average error that had been used previously in our laboratory (Gliner et al., 1981). In this procedure, the subject produced his own standard volume. 
(When the standard volume was provided by the investigator, e.g., from a bag of known volume, there was a large positive constant error which we thus avoided.)

In all three parts of the study, the subject inspired from the spirometer a standard tidal breath (starting at the normal endexpiratory lung volume, designated FRC) that was approximately equivalent to $25 \%, 50 \%$, or $75 \%$ of his inspiratory capacity; the level was specified by the experimenter. (These volumes averaged $884,1,566$, and $2,357 \mathrm{ml}$, respectively.) After the standard breath, the subject exhaled to a level specified by the experimenter, and initiated the reproduction breath from that level, also according to the experimenter's instructions.

In the first part of the study (see Figure 1.1), the subject expired to his normal end-expiratory position and tried to match the standard (volume-FRC reproduction). In the second part of the study (see Figure 1.2), he expired to a level well below the normal end-expiratory volume, to near the residual volume, and then tried to match the tidal volume of the standard breath [volume-RV(residual volume) reproduction]. In the third part of the study (see Figure 1.3), he expired to near residual volume and tried to match the end-inspired position of the standard breath (position reproduction).

In all parts of the study, if the size of the subject's standard deviated by more than $\pm 200 \mathrm{ml}$ from the desired volume, the subject was instructed that his standard was too large or too small. However, no information regarding the reproduction volume was given. The subject was instructed to inspire both the standard and comparison volume at the same inspiratory flow and to maintain it at the same rate throughout the experiment. Inspired flow was not, however, actively regulated by the investigators. Because of changes in respiratory mechanics caused by alteration of end-expired volume prior to production of the comparison volume, a constancy of inspired flow between trials was difficult to obtain. Furthermore, the inspired flow for $25 \%$ IC was typically less than for $75 \% \mathrm{IC}$, since the larger inspired volumes could be attained more comfortably with a more rapid inspired flow.

Each subject participated in all phases of the study; each performed the volume-FRC test first, as it was part of our initial study (Gliner et al., 1981) from which these data were derived (the means differ because only 7 of 14 subjects were included in the present study), but the order of volume-RV reproduction and position reproduction was randomized. The subjects reported to the laboratory on five separate occasions for each part (a total of 15 occasions for the three conditions) of the study. On each occasion, the subject performed 30 test breaths (10 each of $25 \%$, $50 \%$, and $75 \%$ IC, presented randomly). Thus, the average value for each subject was taken over 50 trials for each breath volume under each condition, for a grand total of 450 trial breaths.

\section{Apparatus}

A water-filled spirometer (W. E. Collins) fitted with a 7-liter plastic bell was used in this study. $\mathrm{A} \mathrm{CO}_{2}$-absorbent canister (Sodasorb) was used to remove carbon dioxide. Oxygen was added from a tank of $100 \%$ oxygen to compensate for oxygen utilization. A blower was used to circulate the air within the spirometer, and the bell was weighted so as to compensate exactly for the tendency of the blower to lift the bell. This procedure was chosen to eliminate the need for repeatedly removing and reinserting the mouthpiece. The spirometer bell and the recording kymograph were hidden from the subject's view by an opaque screen.

\section{RESULTS}

For each subject, the mean constant error (mean algebraic error) and the JND (standard deviation of individual constant errors $\times$.6745) were deter- mined for each condition. (In order to make comparisons with other studies in which JNDs have been determined, the standard deviation of the error scores was multiplied by .6745 , yielding the probable deviation, as suggested by Woodworth and Schlosberg, 1965). 1,2 The error of reproducing the test volume was calculated as shown in Figure 1. For parts 1 and 2 , the error was simply the difference between the standard and the reproduction volume. Volumes were not corrected to BTPS (body temperature, ambient pressure, saturated); if this had been done, the volume values would have increased by approximately $10 \%$.

A two-factor repeated-measures analysis of variance was performed on both mean constant error and JND. The two factors were type of reproduction (three levels) and size of breath (three levels). When significant main effects or significant interactions were observed, a Newman-Keuls post hoc test was performed to determine where significant differences lay. An $\alpha$ level of .05 was established a priori.

The results of the volume (FRC) reproductions have been reported previously (Gliner et al., 1981) and were included in the present results for comparison.

Figure 2 shows the mean JNDs and their standard error for the three different treatment conditions at each of the three inspired volumes. Significant main effects were found for respiratory treatment conditions $[F(2,12)=4.70]$ and for inspired volume $[F(2,12)=7.75]$. More important, a significant respiratory treatment $x$ inspired volume interaction was obtained $[F(4,24)=8.51]$. Simple main effects analyses showed significant differences at each volume level (for $25 \%, 50 \%$, and $75 \%$, respectively, Fs $(2,24)=3.95,6.62$, and 20.76). At the $25 \%$ inspired volume, the JND for the position reproduc-

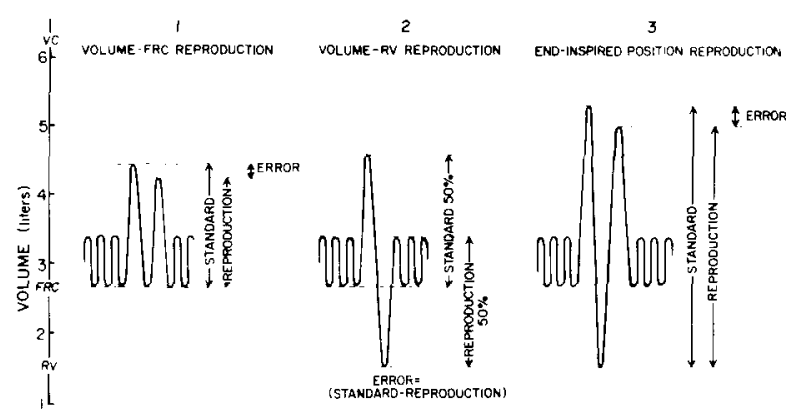

Figure 1. Schematic representation of the protocol, illustrating the method of calculating the error in each of the three procedures. (See text for details.) The standard lung volumes are also noted on this figure. $\mathrm{VC}=$ vital capacity $=$ maximum volume of air which can be exhaled after a full inspiration; IC $=$ inspiratory capacity $=V C-F R C=$ maximum volume of air which can be inhaled from the resting lung volume (FRC); $R V=$ residual volume $=$ volume of air remaining in the lung after a maximum exhalation. 


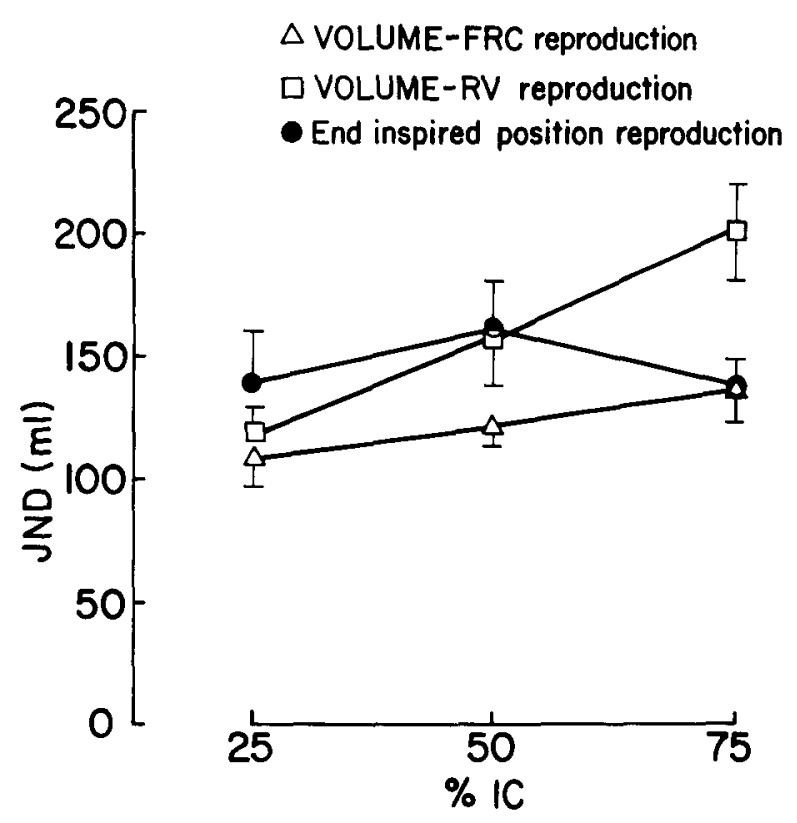

Figure 2. Relationship between just noticeable difference and size of inspired tidal volume. The slope of the line is the Weber fraction: .021 for volume-FRC reproduction (volume + position); .068 for volume-RV reproduction (volume reproduction); -.001 for end-inspired position reproduction. The bars indicate the standard error.

tion condition was significantly larger than that of the volume-FRC condition, but no other significant difference was revealed. At the $50 \%$ inspired volume, the position reproduction condition and the volume$R V$ reproduction condition did not differ from each other but were significantly greater than those of the volume-FRC reproduction condition. At the $75 \%$ inspired volume, the volume-RV reproduction condition was significantly larger than either position reproduction or volume-FRC reproduction conditions, which did not differ significantly from each other.

Significant simple main effects were also obtained within respiratory treatment conditions, but only for the volume-RV reproduction condition $[F(2,24)=$

Table 1

Constant Error in Reproduction of Inspired Volume (in Milliliters) or Inspired Position

\begin{tabular}{|c|c|c|c|c|c|c|}
\hline & \multicolumn{2}{|c|}{$\begin{array}{r}\text { Volume-FRC } \\
\text { Reproduction } \\
\end{array}$} & \multicolumn{2}{|c|}{$\begin{array}{l}\text { Volume-RV } \\
\text { Reproduction }\end{array}$} & \multicolumn{2}{|c|}{$\begin{array}{c}\text { Position } \\
\text { Reproduction }\end{array}$} \\
\hline & Mean & SD & Mean & $\mathrm{SD}$ & Mean & SD \\
\hline $\begin{array}{l}25 \% \mathrm{IC} \\
50 \% \mathrm{IC} \\
75 \% \mathrm{IC}\end{array}$ & $\begin{array}{r}65 \\
7 \\
-27\end{array}$ & $\begin{array}{r}69 \\
105 \\
88\end{array}$ & $\begin{array}{l}251 \\
215 \\
215\end{array}$ & $\begin{array}{l}131 \\
-\quad 123 \\
251\end{array}$ & $\begin{array}{r}-110 \\
-89 \\
-77\end{array}$ & $\begin{array}{l}281 \\
165 \\
225\end{array}$ \\
\hline
\end{tabular}

Note-These SDs were not used to calculate JND. Volume-RV is greater than the other procedures. IC = inspiratory capacity (see legend for Figure 1).
29.04]. Within this condition, the JND increased significantly at each increase in inspired volume. Therefore, the JND data show that, at the smallest inspired volume, the lack of volume cues produced a significant decrease in accuracy of position reproduction, whereas a lack of position cues did not significantly decrease accuracy of volume reproduction. At the middle inspired volume, the lack of either volume or position cues decreased accuracy significantly and similarly. At the largest inspired volume, the lack of position cues decreased volume reproduction accuracy significantly, whereas the lack of volume cues did not affect position reproduction. Also, accuracy in reproducing volume when position cues were absent (volume-RV condition) decreased as the size of the inspired volume increased.

The constant error (Table 1) was also analyzed for each respiratory treatment at each inspired volume in order to determine in which direction the subjects were in error. Significant main effects were observed for respiratory treatment condition $[F(2,12)=9.62]$. The Newman-Keuls post hoc test showed that the volume-RV reproduction condition yielded significantly larger constant errors than either the volume-FRC reproduction condition or the position reproduction condition, with the latter two not differing significantly from each other. There were no other significant main effects or significant interaction effects for this measure. Therefore, it appeared that the lack of position cues caused the subjects to overestimate the reproduction volume consistently, regardless of the size of the standard inspired volume.

\section{DISCUSSION}

The sensory information that enables an individual to reproduce the size of an inspired breath appears to be dependent upon the lung volume (Gliner et al., 1981). In the present study, our aim was to determine the importance of a relatively constant resting endexpired lung volume (FRC) and how changing the typical end-expired volume would alter a subject's ability to reproduce inspired volume.

Our results demonstrated that reproduction of inspired volume was, in general, more precise when end-expired volume was maintained at a relatively constant level.

At the smallest size inspiration that we tested, $25 \%$ IC (mean $=880 \mathrm{ml}$ ), the subjects reproduced the inspired volume quite accurately despite a reduction in FRC of more than $1,000 \mathrm{ml}$. However, since they were somewhat less successful at reproducing the end-inspired volume under the same conditions, some cue other than end-expired volume must be used in reproducing volume when the volume is small. The most likely mechanism is that 
an "impulse-timing" method is used to reproduce small tidal volumes (i.e., the sense of effort and duration of effort are gauged and then reproduced, without regard to initial or final absolute lung volume).

When the inspired standard volume was at $\mathbf{7 5 \%}$ IC, reproduction of end-inspired volume was more accurate than reproduction of tidal volume. This difference suggests that the cue of end-inspired volume may be important in reproducing large, but not small, tidal volumes. It is not clear what receptors provide this sensory information. DiMarco, Gottfried, Wolfson, and Altose (1981) studied volume perception in normal and quadriplegic subjects and found similar exponents for magnitude estimation of tidal volume in both groups. This suggests that neither joint receptors nor receptors located in the skin provide the sensory information. Furthermore, the similarity of the exponent in both passive and active ventilation indicates that sensation of muscle force was not a factor in the overall ability to perceive volume changes. Halttunen (1974), however, found a significant correlation between intercostal electromyogram impulse frequency and estimation of inspired volume. Such a correlation does not necessarily imply a contradiction between these two studies, but it is important, in that the quadriplegics in the study of DiMarco et al. (1981) had no intercostal muscle innervation. Nevertheless, these investigators did not completely rule out some role for chest wall receptors in detecting respiratory loads.

The measure of precision of reproduction, the JND, is derived from a measure of variability, the standard deviation. But the JND does not take into account the accuracy of reproduction, since it does not reflect the presence of a systematic constant error. In the tidal volume reproductions, there was a large positive constant error, which shows a clear tendency of the subjects to overestimate tidal volume when the initial, end-expired, volume is reduced. Clearly, the error was in the direction of the endinspired position of the standard breath. This finding is consistent with those of similar studies of the accuracy of reproduction of finger movements (Kelso, Holt, \& Flatten, 1980), in which subjects moved their index fingers a given distance and then attempted to match the distance moved. When the starting position was changed between the standard and reproduction movement, the latter tended to be larger than the standard movement when the starting point was moved farther away from the endpoint of the previous movement (i.e., reproduction was biased in the direction of the end position of the standard movement). However, the apparent similarity between the present results and those of finger-movement studies may be misleading. The positive constant error in volume- $R V$ reproduction could also result from the change in lung-chest wall mechanics when the end-expired volume is reduced. This change results in a component of chest wall elastic recoil which would tend to produce an increase in lung volume and thus may be responsible for the volume overshoot, but which did not result in an increased variability of reproduction as measured by the JND. Halttunen (1974) observed that when the respiration of his subjects was stimulated by breathing $5 \% \mathrm{CO}_{2}$ during magnitude estimation of inspired volume, they produced a larger volume at any given estimated magnitude than they did during air breathing. His finding suggests that the increased chemical drive to ventilation by $\mathrm{CO}_{2}$ may modify the perception of lung volume. In our experimental task, a near full expiration between the standard and reproduction breath would probably cause a small increased drive to breathing.

The ability to reproduce absolute end-inspired volume is apparently unrelated to the tidal volume of the standard breath. Perceptual continua may be described as prothetic (dealing with judgments of magnitude-“'how much"), which typically subscribe to the psychophysical power law, or metathetic (dealing with quality and/or location of sensation-"what kind" and "where"), which do not. The larger intercept (threshold) and flat slope indicate that position reproduction does not subscribe to Weber's law, which may indicate that these position or location cues do not have a magnitude, as such, and thus may be characterized as lying on a metathetic continuum (Stevens, 1975). On the other hand, the reproduction of inspired volume does subscribe to Weber's law in the present study and has been shown to subscribe to the psychophysical power law in other studies (Halttunen, 1974), observations which suggest that inspired volume is a prothetic continuum.

The question of whether it is easier to reproduce volume magnitude or volume position cannot be answered unequivocally by these data. In the absence of a constant initial volume, both magnitude and position reproduction are impaired. These studies do not provide definitive information on which receptor modalities may be involved in perceiving volume changes.

\section{REFERENCES}

Dimarco, A. F., Gottraied, S. B., Wolf8on, D. A., \& Attose, M. D. Perception of inspired volume in normal and quadriplegic subjects. American Review of Respiratory Disease, 1981, 123(4, Part 2: 202).

EDWARDs, A. Statistical methods for the behavioral sciences. New York: Holt, Rinehart \& Winston, 1964.

Gliner, J. A., Folinsbee, L. J., \& Honvath, S. M. Accuracy and precision of matching inspired lung volume. Perception \& Psychophysics, 1981, 29, 511-515. 
HaltTUNen, P. K. The voluntary control in human breathing. Acta Physiologica Scandinavica, 1974, Supplement 419.

Kelso, J. A. S., Holt, K. G., \& Flatt, A. E. The role of proprioception in the perception and control of human movement: Toward a theoretical assessment. Perception \& Psychophysics, 1980, 28, 45-52.

Stevens, S. S. Psychophysics. New York: Wiley, 1975.

Woodworth, R. S., \& Schlosberg, H. Experimental psychology. New York: Holt, Rinehart \& Winston, 1965.

\section{NOTES}

1. In the classical method of average error, the measure of discrimination is typically the standard deviation of the compari- son value. If the standard is a constant, it is immaterial whether the standard deviation of the comparison value or of the difference score (i.e., standard-comparison) is used. However, since our standard was not constant (i.e., the subject produced his own standard volume), we were forced to use the standard deviation of the difference score as a measure of discrimination.

2. In a normal distribution, the interval established by the mean, plus or minus one probable deviation, contains the middle $50 \%$ of the measures (Edwards, 1964), which corresponds to the difference limen from the method of limits (Woodworth \& Schlosberg, 1965, pp. 199-210).

(Manuscript received February 11, 1982;

revision accepted for publication September 3, 1982.) 